\title{
Growth Hormone Day Curve (GHDC) in the management of patients with Acromegaly. Can we cut down on the cost?
}

\author{
Ahmad ANM1, Mohamed RS1, Tennakoon SUB², Antonypillai CN1 \\ ${ }^{1}$ Diabetes and Endocrine Unit, National Hospital Kandy, Kandy, Sri Lanka \\ 2Department of Community Medicine, Faculty of Medicine, University of Peradeniya, Peradeniya, Sri Lanka
}

Abstract

\begin{abstract}
Background
Acromegaly is a clinical syndrome resulting from hypersecretion of Growth Hormone (GH). Biochemical tests used in managing Acromegaly include for GH, IGF-1, random GH and GHDC. GHDC is useful to assess clinical severity and response to treatment. This test is usually done as a 5-point test, but there is no widely accepted protocol regarding the timing of samples. Mean GH $<5 \mathrm{mU} / \mathrm{L}$ suggest adequate control of $\mathrm{GH}$.

Objectives

To check if lesser number of samples can be used rather than five-point GHDC and to find the proportion of patients adequately responding to treatment.

Methods

This was a retrospective descriptive study done at Endocrinology department of TH Kandy. GHDC data were retrieved from clinic records and analyzed using Medcalc2019.

Results

Fifty-eight GHDCs of 30 patients were included in the final analysis. We compared 14 different combinations of four and three samples with the established way of 5-point sampling. All combinations showed $100 \%$ sensitivity in detecting adequate control while maintaining a specificity of 95 to $100 \%$. There were 5 false positives where means ranged from 5.08 to 6.52 suggesting biochemically mild disease. Only one third of our patients had adequately controlled disease while most international data show better outcome.

Conclusions.

Traditional GHDC may safely be replaced with a 4-point/3-point test while maintaining $100 \%$ sensitivity and slightly compromised specificity. There is a need to produce local data and to make advanced treatment modalities available in the public sector to improve patient outcome.
\end{abstract}

Keywords: Acromegaly, Growth, Hormone, Day, Curve

Correspondence email: muneeranm@yahoo.com

ORCID ID: https://orcid.org/0000-0002-6719-1855

Copyright: This is an open-access article distributed under the terms of the Creative Commons Attribution License, which permits unrestricted use, distribution, and reproduction in any medium, provided the original author and source are credited (CC BY 4.0)

\section{Introduction}

Acromegaly is an important endocrine disorder encountered by Endocrinologists in clinical practice. Apart from increasing morbidity and mortality, Acromegaly has a significant negative impact on quality of life. This is a clinical syndrome resulting from hyper secretion or increased availability of Growth Hormone (GH). Although GH has some direct actions, majority of its effects are brought about by the production of Insulin like Growth Factor 1 (IGF1). The causative lesion is a pituitary adenoma in more than $95 \%$ of cases, of which almost three fourths are due to macroadenomas. ${ }^{(1,2)}$
Biochemical tests used in the management of patients with Acromegaly include Glucose suppressed GH levels (OGTT$\mathrm{GH})$, IGF1, random $\mathrm{GH}$ and mean $\mathrm{GH}$ from a Growth Hormone Day Curve (GHDC). In spite of not being included currently in major guidelines, GHDC is still included in some hospital-based protocols ${ }^{(3,4)}$. Further, there are certain issues related to the use of other tests mentioned above. For instance, IGF-1 test is known to show significant inter-assay variability and IGF-1 level is affected by several conditions other than Acromegaly $(6,7)$. For OGTT-GH, patients need to fast and the need to take a glucose solution can be very nauseating to some 
patients. Random GH is one of the tests used for monitoring in patients undergone or undergoing some types of treatment as a level of $<3 \mathrm{mU} / \mathrm{L}$ is shown to correlate with the control of Acromegaly(2). In addition to the pulsatility of GH secretion, it has been shown that there is a significant increase in the fluctuation of GH levels among patients in whom adequate disease control is achieved through surgery $(8)$. Since GH levels in normal individuals can fluctuate from 'undetectable' up to $90 \mathrm{mU} / \mathrm{L} 1$, a single random $\mathrm{GH}$ is not used for diagnostic purposes. Measurement of $\mathrm{GH}$ is also associated with significant inter-assay variability1. More importantly, most of the available data on prognosis of Acromegaly is based on mean GH or random GH but, not on IGF-1 or post-OGTT nadir GH8. Karavitaki et al. in 2007, after their analysis of data from OGTT, GHDC and IGF-I done in 26 post-surgical patients with Acromegaly concluded 'Reluctantly, in cases of Acromegaly following adenomectomy, the OGTT and the GHDC do not always provide consistent data making both tests necessary for the assessment of the therapeutic result $(9)$.

GHDC is useful to assess clinical severity of growth hormone excess in Acromegaly and to assess response to treatment. In addition, pre-treatment mean GH has been shown to be a major determinant of outcome of surgery8. GH should normally be $<1 \mathrm{mU} / 1$ on at least 2 of the samples, but a mean $\mathrm{GH}<5 \mathrm{mU} / \mathrm{L}$ in the growth hormone day curve suggest adequate control of GH in patients with treated Acromegaly 3 , 4,9. This test is usually done as a 5 -point test, but there is no widely accepted protocol and there are differences among different institutions with regard to the number and timing of samples $^{3}, 4,5$. In our center; The Endocrinology Unit of National Hospital, Kandy, Sri Lanka; sampling is done at 9:00 AM, 11:00 AM, 1:00 PM, 3:00 PM and 5:00 PM considering the availability of resources. GHDC does not require any special preparations apart from IV cannulation. Patients can eat and drink normally and are allowed to take their usual medications. The drawbacks of this procedure include cost and time consumption. So, it is important to identify, if any, modifications that can be made to GHDC in order to overcome these issues and still obtain a better result compared to 'single random GH'.

The main objective of our study was to assess the efficacy of less than 5 measurements of GH level compared to the fivepoint testing during GHDC. Our other objective was to find out the percentage of our acromegaly patients whose disease was adequately controlled with the treatment.

\section{Methodology}

This was a retrospective study carried out at the Diabetes and Endocrine unit, National Hospital Kandy, Sri Lanka. Patients, who have had a diagnosis of Acromegaly due to a pituitary adenoma and undergone either one or a combination of surgery, medical therapy and radiotherapy and followed up at Diabetes and Endocrine unit, National Hospital Kandy, was the study population. Their demographic details and GHDC data were retrieved from records maintained in the clinic. The inclusion was all patients with Acromegaly due to a pituitary adenoma who had received at least one mode of treatment at the time of data collection for the study. Patients with Acromegaly due to any cause other than a pituitary adenoma and those with Acromegaly who had not received any treatment were excluded. All patients whose data was available at the clinic and met inclusion and exclusion criteria were included in the study. Data were retrieved from patients' clinic record and were analyzed using mono-variate and bi-variate analysis with the help of the Medcalcsoftware (2019 MedCalc Software).

\section{Ethical considerations:}

This was a study involving the analysis of anonymized data which was already available at the unit. Therefore, obtaining consent from the individual patients was not considered necessary. Research proposal was submitted for review by Ethical review committee of Faculty of Medicine of University of Peradeniya. It was exempted from the need for ethical review due to the nature of the study. This study has the potential to help cut down on the number of sampling and therefore reduce the number of tests and the time consumed.

\section{Results}

Sixty GHDC data were retrieved from a total of 31 patients with Acromegaly. Due to missing data with 2 curves, 58 GHDCs of 30 patients were included in the final analysis. Given the fact that majority of patients (16/30) had more than one GHDCs done on them, we considered the latest curve to assess the control of Acromegaly.

Using the criteria of mean $\mathrm{GH}<5 \mathrm{mU} / \mathrm{L}$ to suggest adequate control of GH secretion, $33 \%(10 / 30)$ of our patients had adequate control of Acromegaly. Going by the stricter criteria of $<1 \mathrm{mU} / 1$ on at least 2 of the samples, only half $(5 / 10)$ of these patients had evidence of presumed 'normal' $\mathrm{GH}$ secretion.

To find out whether lesser number of samples can be used rather than five during GHDC with reasonable specificity, we compared 14 different combinations of four and three samples with the established way of five samples (Gold standard test). Among the total of 58 GHDCs, mean GH was $<5 \mathrm{mU} / \mathrm{L}$ in 18. All the combinations tested showed $100 \%$ sensitivity (i.e. Zero false negatives) in detecting adequate control. In addition, 3 out of 4 possible 4 -point combinations showed 100\% specificity as well whereas the remaining one gave only a single false positive finding (Specificity 97.5\%, see Table-1 below). Specificities of 3-point testing ranged from 95 to $100 \%$. 
Table 1 Performance of different 3-and 4-point combinations in comparison to the gold standard 5-point testing.

\begin{tabular}{llllll}
\hline & Sample timing & Sn\% & Sp\% & PPV $\%$ & NPV\% \\
\hline 4-point combinations & $1,2,3,4$ & 100 & 100 & 100 & 100 \\
& $1,2,3,5$ & 100 & 97.50 & 95 & 100 \\
$1,3,4,5$ & 100 & 100 & 100 & 100 \\
3-point combinations & $2,3,4,5$ & 100 & 100 & 100 & 100 \\
& $1,2,3$ & 100 & 97.50 & 95 & 100 \\
& $1,2,4$ & 100 & 97.50 & 95 & 100 \\
$1,2,5$ & 100 & 100 & 100 & 100 \\
$1,3,4$ & 100 & 100 & 100 & 100 \\
$1,3,5$ & 100 & 97.50 & 95 & 100 \\
& $1,4,5$ & 100 & 97.50 & 95 & 100 \\
$2,3,4$ & 100 & 95 & 90 & 100 \\
$2,3,5$ & 100 & 95 & 90 & 100 \\
$2,4,5$ & 100 & 95 & 90 & 100 \\
& $3,4,5$ & 100 & 95 & 90 & 100 \\
\hline
\end{tabular}

$\mathrm{Sn}=$ Sensitivity, $\mathrm{Sp}=$ Specificity, $\mathrm{PPV}=$ Positive predictive value, $\mathrm{NPV}=$ Negative predictive value, 1=9:00 AM, 2=11:00

AM, 3=1:00 PM, 4=3:00 PM and 5=5:00 PM.

Overall, there were 5 false positive results when using a3point or 4-point combination in interpreting the total of 58 GHDCs $(8.6 \%)$. On analyzing these 5 GHDCs, it was evident that despite the fact that the overall mean did not suggest adequate control of the disease, the actual means were just outside the normal range and ranged from 5.08 to 6.52. This indicates that if we use a 3-point or 4-point test, and the result shows adequate suppression, there is an $8.6 \%$ probability of it being an uncontrolled disease albeit biochemically mild. This may still be important to be aware of since even mildly abnormal mean GH has been shown to be associated with increased morbidity and mortality. Therefore, exercising caution while interpreting marginal values and consideration of clinical features together with other test results such as IGF-1 will help to prevent wrong decision making.

\section{Discussion}

Acromegaly is associated with increased morbidity and mortality unless complete remission or adequate biochemical control is achieved. Biochemical confirmation of Acromegaly is fairly straightforward and is based on elevated IGF-1 along with failure to adequately suppress GH levels during a 75-g OGTT. Nevertheless, there is difference of opinion with regards to the assessment of disease severity. GHDC is one of the tests among others used in patients with Acromegaly to assess the severity and the response to various treatment modalities. Despite producing useful information, this is not widely performed nowadays mainly because it is a time consuming and labour intensive investigation. Therefore, we aimed to investigate whether a less than 5 -point testing would give comparable results to 5-point testing8.

Our data shows that 3-point and 4-point GHDC has 100\% sensitivity along with 95 to $100 \%$ specificity. There is an $8.6 \%$ risk of getting false positive result. We could not detect any particular time point which made false positive result more likely. Therefore, we propose that the conventional 5-point GHDC can be safely replaced with a 3-point or 4-point GHDC with samples taken

Anytime during the day while maintaining a gap of 2 hours between the samples.

According to the GHDC cutoff of $<5 \mathrm{mU} / \mathrm{L}, 33 \%$ of Acromegaly patients treated at our unit were rendered adequately controlled. This rate of successful biochemical control is less compared to most of the published data. With experienced pituitary surgeons, endoscopic 
transsphenoidal microsurgery has been shown to result in an initial remission rate of $85 \%$ for microadenomas and 40 $-50 \%$ for macroadenomas ${ }^{10}$.

There are multiple possible reasons for the less favourable outcome for our patients. Our patients still tend to seek medical advice late having had morphological changes ignored or missed for a long time. This means that they are more likely to harbor larger macroadenomas and therefore cavernous invasion which precludes success from neurosurgical intervention. Further, during work up and planning there are delays and waiting times since at least some of these hormonal tests and transsphenoidal surgeries done in limited number of centres. Sometimes, there is difficulty in getting patients' consent for surgeries and even radiotherapy which delays the treatment. There is also limited availability or unavailability of certain treatment options. For example, stereotactic radiosurgery (SRS) has become available only very recently in Sri Lanka, thus most if not all of the patients in our study would not have had the option of getting SRS. Further, long acting somatostatin analogues are not freely available and Pegvisomant is not yet registered in the country. Finally, because of the cost of the investigations and treatments, most of the patients cannot afford to get these done in the private sector.
Total population of our country being about 22 million, we will not see many patients with a rare condition like Acromegaly. Most of the research data available to us is from the western or the developed world. Knowing the fact that there are environmental, genetic, and cultural and other differences, it may not be the ideal thing to extrapolate these research data to manage our patients. This strongly argues for the importance of a national registry and local research on Acromegaly and conditions alike to enable us to generate our own data and to identify deficiencies in the system where we can improve.

\section{Conclusion}

GHDC is one of the biochemical tests available for managing patients with Acromegaly. If this is to be used, our data indicate that the traditional 5-point test can be safely replaced with a 4-point or 3-point testwhile maintaining $100 \%$ sensitivity but with a slight compromise on specificity. Treatment success rate for our patients with Acromegaly lags behind most of the treatment outcomes among published data. Steps should be taken to make the more advanced treatment options available in the government sector. There is a need to produce our own data to identify other aspects of patient management on which we can improve to achieve a better outcome for our patients.

\section{References}

1. Melmed S, Kleinberg D.Pituitary Masses and Tumors, Williams's text book of endocrinology. 13th edition: Saunders Elsevier. 2016:229-323

2. Katznelson et al. Acromegaly Clinical Practice Guidelines. Journal of Clinical Endocrinology and Metabolism, 2014;99(11):3933-395

3. BartsEndocrine e-protocol. Acromegaly and Growth Hormone. 2009

4. Hayden K. Growth Hormone Day Curve. Version-3. Q-Pulse CB-CLIN-PRO-030. 2017

5. Norfolk and Norwich University Hospitals Foundation Trust Growth Hormone Day Curve protocol. Endobible 2018

6. Bidlingmaier M, Friedrich N, Emeny RT, et al. Reference intervals for insulin-like growth factor-1 (IGF-1) from birth to senescence: results from a multicenter study using a new automated chemiluminescence IGF-I immunoassay conforming to recent international recommendations. Journal of Clinical Endocrinology and Metabolism. 2014; 99:1712-1721.

7. Frystyk J, Freda P, Clemmons DR. The current status of IGF-I assays-a 2009 update. Growth Horm IGF Res. 2010;20:8 -18

8. Kaltas G A. Predictors of the Outcome of Surgical Treatment in Acromegaly and the Value of the Mean Growth Hormone Day Curve in Assessing Postoperative Disease Activity. The Journal of Clinical Endocrinology \& Metabolism. . 2001;86( 4):1645-52

9. Niki Karavitaki, Alberto Fernandez, Violet Fazal-Sanderson, Helen E Turner \& John AH Wass. Evaluation of acromegaly status following surgery by OGTT, GH day curve andIGF-I:relationships between these parameters. Endocrine Abstracts 2007; 13 :225 